\title{
PÓS-OPERATÓRIO DE PACIENTES COM CÂNCER COLORRETAL ESTOMIZADOS: UMA ANÁLISE COMPREENSIVA
}

\author{
Postoperative period of ostomized patients with colorectal cancer: a comprehensive analysis \\ Post operatorio en las personas con cáncer colorrectal estomizadas: un análisis comprensivo
}

\author{
Suellen Cristina Rodrigues', Selme Silqueira de Matos², Aidê Ferreira Ferraz², Miguir Terezinha Vieccelli Donoso ${ }^{3}$, \\ Eline Lima Borges ${ }^{4}$, Salete Maria de Fátima Silqueira ${ }^{5}$, Adelaide De Matti', Daniela Mascarenhas de Paula ${ }^{6}$
}

RESUMO: Objetivo: Compreender os sentimentos da pessoa colostomizada devido ao câncer colorretal. Métodos: Os relatos dos entrevistados foram transcritos e analisados sob o referencial teórico da análise de conteúdo, na sua modalidade temática. Os pacientes entrevistados estavam internados nas clínicas de coloproctologia e oncologia dos ambulatórios de um hospital de grande porte de Belo Horizonte, Minas Gerais. Resultados: Emergiram duas categorias: "conviver com sentimentos relacionados ao estoma e enfrentá-los" e "influências nas concepções sobre a vivência da sexualidade e do relacionamento conjugal", que foram divididas em subcategorias. Conclusão: Sugere-se uma mudança de perspectiva quanto ao cuidado dos participantes deste estudo, priorizando a capacitação de profissionais que lidam com essas pessoas, para que sejam assistidas de forma integrada e humanística, minimizando-se as dificuldades enfrentadas pelo paciente e pelos profissionais de saúde.

Palavras-chave: Colostomia. Oncologia. Resiliência psicológica. Sexualidade.

ABSTRACT: Objective: To understand the feelings of ostomized patients with colorectal cancer. Methods: Reports of interview respondents were transcribed and analyzed under the theoretical framework of content analysis in its thematic modality. Patients interviewed were hospitalized in the coloproctology and oncology clinics of a large hospital in Belo Horizonte, Minas Gerais, Brazil. Results: Two categories emerged: "coping with and facing feelings related to the stoma" and "influences on the visions of sexuality and marital relationship". These were then divided into subcategories. Conclusion: a change of perspective on the care of these patients is suggested, with focus on training of professionals who deal with them, so they are assisted in an integrated and humanistic way, thus minimizing difficulties faced by both the patient and the health professional.

Keywords: Colostomy. Medical oncology. Resilience, psychological. Sexuality.

RESUMEN: Objetivo: Comprender los sentimientos de los pacientes de cáncer a convertirse en estomizado. Métodos: Los informes de los encuestados fueron transcritos y analizados bajo el análisis de contenido teórico, en su modalidad temática. Los pacientes entrevistados fueron hospitalizados en clínicas de cirugía colorrectal y en clínicas de oncología de un hospital en Belo Horizonte, Minas Gerais, Brasil. Resultados: Emergieron dos categorías, "vivir con sensaciones relacionadas con el estoma y hacerles frente" y "influencias de las concepciones de la experiencia de la sexualidad y relación matrimonial", divididas en subcategorías. Conclusión: Se sugiere un cambio de perspectiva en la atención de los participantes en el estudio, dando prioridad a la formación de profesionales que se ocupan de estas personas, que pueden ser asistidos de manera totalmente paga y humanista, reduciendo al mínimo las dificultades sufridas por el paciente y por los profesionales de la salud.

Palabras clave:Colostomía. Oncología médica. Resiliencia psicológica. Sexualidad.

\footnotetext{
'Graduada em Psicologia, especialista em Psico-oncologia; Psicóloga do Hospital Felício Rocho. E-mail: suellen.psique@yahoo.com.br

2Doutora em Enfermagem; Docente da Escola de Enfermagem da Universidade Federal de Minas Gerais (UFMG). E-mail: selmesilqueira@gmail.com; afferraz@terra.com.br

${ }^{3}$ Doutora em Ciências da Saúde; Docente da Escola de Enfermagem da UFMG. E-mail: miguirdonoso@uol.com.br; adelaidedemattia@gmail.com

Escola de Enfermagem da Universidade Federal de Minas Gerais. Avenida Alfredo Balena, 190 - Campus Saúde - Santa Efigênia - CEP: 31130-100 - Belo Horizonte (MG), Brasil.

${ }^{4}$ Doutora em Enfermagem Fundamental; Docente da Escola de Enfermagem da UFMG. E-mail: eborges@ufmg.br

${ }^{5}$ Doutora em Saúde Pública; Docente da Escola de Enfermagem da UFMG. E-mail: saletesilqueira@gmail.com

${ }^{6}$ Mestre em Enfermagem; gerente de Enfermagem do Hospital Felício Rocho. E-mail: danielamascarenhas1@gmail.com

Recebido: 20 abr. 2016 - Aprovado: 24 jun. 2016

DOI: $10.5327 / Z 1414-4425201600020005$
} 


\section{INTRODUÇÃO}

O câncer colorretal (CCR) é o terceiro câncer mais comum e a terceira principal causa de morte por câncer em homens e mulheres nos Estados Unidos da América. Quase dois terços dos sobreviventes de CCR vivem em torno de cinco anos após o diagnóstico ${ }^{1}$. Em alguns países desenvolvidos esse é o segundo tipo de câncer mais comum em termos de incidência. Em várias partes do mundo apresenta incidência e mortalidade crescentes ${ }^{2}$.

Muitos procedimentos cirúrgicos para eliminar o câncer podem resultar na confecção de um estoma intestinal, sendo os mais frequentes a colostomia e a ileostomia. Esses estomas têm por objetivo promover a eliminação de efluentes e flatos para o meio externo, sendo, portanto, denominados estomas de eliminação ${ }^{3}$.

A condição de se estar estomizado por câncer conduz a situações que fogem às normas culturais e afetam os significados que a pessoa dá a si mesma, à doença, ao tratamento e à própria existência ${ }^{4}$. O diagnóstico de câncer leva o sujeito a adentrar no mundo da doença e dos tratamentos e, com o estoma, seu corpo muda juntamente com sua existência 5 . Lembra-se de que, muitas vezes, a pessoa passa a conviver com duas realidades concomitantes: ter um diagnóstico de câncer e, em função da propedêutica, ter um estoma de eliminação. Os profissionais de saúde devem considerar essas questões no planejamento da assistência.

A pessoa, ao receber informações sobre o diagnóstico da doença e a necessidade de realização de uma colostomia, vivencia dificuldades de enfrentar e vencer as múltiplas mudanças que ocorrerão nessa nova fase da vida, incluindo pré, trans e pós-operatório ${ }^{6}$. Vivencia também significativas alterações na dinâmica sócio-familiar. Na maioria das vezes percebe-se que os colostomizados se apresentam fragilizados devido à nova situação em que se encontram ${ }^{7}$.

$\mathrm{Na}$ atuação da equipe multidisciplinar do hospital onde ocorreu esta pesquisa, principalmente no que tange ao psicólogo e ao enfermeiro, esses profissionais percebem que pacientes oncológicos colostomizados se encontram em dupla situação de vulnerabilidade: o enfrentamento de uma doença estigmatizada como o câncer e a consequente condição de ser estomizado. Essa situação se agrava quando a colostomia é permanente. As reações variam desde vergonha, dificuldade de verbalização, ansiedade, sentimento de culpa e decréscimo da autoestima, entre outras 8 .

A assistência multidisciplinar tem por objetivo a reabilitação, a qual envolve uma avaliação compreensiva, levando-se em conta a subjetividade de cada pessoa assistida. Lembra-se de que a assistência ao paciente cirúrgico demanda, além dos cuidados com o físico, apoios social, espiritual e emocional ${ }^{9}$.

A condição de fragilidade dessas pessoas demanda maior apoio dos profissionais envolvidos. No entanto, nem sempre nesse processo os sentimentos vivenciados pelo paciente com colostomia decorrente do câncer são relevados pelo psicólogo ou pelo enfermeiro em seu cotidiano assistencial, o que se considera o problema desta pesquisa. Há necessidade de uma abordagem compreensiva, tendo-se em vista questões subjetivas que se delineiam a partir do acometimento da doença e, posteriormente, a partir da confecção da colostomia.

\section{OBJETIVO}

Compreender os sentimentos da pessoa colostomizada devido ao CCR.

\section{MÉTODO}

Trata-se de estudo qualitativo, de natureza exploratória, realizado em hospital privado de grande porte de Belo Horizonte, Minas Gerais. Nessa instituição são atendidos pacientes de diversas especialidades clínicas e cirúrgicas, incluindo coloproctologia, nas unidades de internação, terapia intensiva, pronto atendimento e ambulatorial. São assistidos pacientes de diversas faixas etárias.

A pesquisa contou com amostra por conveniência. Foram definidos como critérios de inclusão: pacientes maiores de 18 com colostomia definitiva confeccionada há mais de três meses devido ao CCR em estágio III (o câncer invade linfonodos vizinhos, mas não outras partes do corpo) ou IV (câncer já pode ser encontrado a distância do local original), que se encontravam internados para tratamento das complicações da doença ou do estoma,e concordaram em participar da pesquisa, assinando o Termo de Consentimento Livre e Esclarecido (TCLE).

A população foi constituída por 18 pacientes com colostomia ou ileostomia, e a amostra, por 5 colostomizados que atenderam aos critérios de inclusão.

Para a coleta de dados utilizou-se a entrevista semiestruturada, pois esta permite caracterizar a subjetividade e a particularidade dos discursos. Teve como base três questões norteadoras: 
1. Como se sente com o uso da bolsa coletora e de que maneira vem enfrentando essa experiência?

2. Como tem sido o manuseio para lidar com esse dispositivo?

3. Poderia me falar sobre sua sexualidade, após a confecção do estoma?

As entrevistas foram realizadas por uma das pesquisadoras, no período de 30 de janeiro a 02 de março do ano de 2015 , no Consultório de Psicologia da Unidade de Oncologia do hospital deste estudo, garantindo-se a privacidade dos entrevistados. Para preservar o anonimato, as entrevistas foram codificadas em E1, E2, E3, E4 e E5.

Para a análise dos achados, seguiram-se as etapas preconizadas pela análise de conteúdo, na modalidade temática, proposta por Bardin ${ }^{10}$, que recomenda operações de desmembramento de um texto em unidades e categorias, de acordo com reagrupamentos analógicos. Entre as diferentes formas de categorização, a investigação por meio de temas é considerada eficaz e rápida no caso de discursos simples e diretos. Foram seguidas as etapas: pré-análise, exploração do material, tratamento dos dados (inferência e interpretação). Na pré-análise ocorreram a escuta das entrevistas, a transcrição e a leitura flutuante dos achados. Essas ações permitiram a apropriação inicial do conteúdo, com posterior ramificação das partes mais relevantes ao objetivo da pesquisa para a constituição do corpus do trabalho, por meio dos critérios de representatividade, homogeneidade e pertinência. A segunda etapa foi caracterizada por operações de decomposição, consistindo na exploração exaustiva do material visando à consolidação das categorias, por meio do recorte dos núcleos de sentido nas falas dos participantes, que conferiram significado aos aspectos analisados no estudo. Na terceira etapa, os dados selecionados receberam tratamento a fim de serem válidos e significativos.

Destaca-se que a pesquisa recebeu a aprovação do Comitê de Ética e Pesquisa do referido hospital, $n^{\circ}$ CAAE 234853214.8.0000.5125, parecer 888.066, em 29 de outubro de 2014 e que todas as normas da Resolução n 466/12 do Conselho Nacional de Saúde foram seguidas.

\section{RESULTADOS}

Dos cinco sujeitos que constituíram a amostra deste trabalho, três eram mulheres e dois eram homens, quatro estavam casados e um estava divorciado. A faixa etária variou de 53 a
68 anos e a idade média foi de 59 anos. Em relação à profissão, um sujeito era trabalhador do lar, um era bancário, um era costureiro, um era psiquiatra e um era professor.

A seguir, depoimentos que resultaram na formação das duas categorias emergentes, "enfrentamento" e "sexualidade":

[...] eu tinha tanto medo, pois só conseguia dormir de barriga para cima, com medo de virar de lado e a placa soltar e acontecer alguma coisa com ela, ficava insegura, acordava várias vezes na noite com essa preocupação. (E5)

Eu ficava constrangido pelo fato de ser uma coisa inesperada, que aparece, mas com o tempo a gente vai melhorando o sentimento ruim que a gente tem com relação a ela. (E1)

[...] eu fico tão impressionada e tão triste, tão revoltada, fugiu do meu controle, eu não sabia que isso iria acontecer comigo não, Deus me livre, credo [choro]. (E3)

[...] agora estou me acostumando, to aceitando na verdade, to levando uma vida assim, mais perto da normal, tanto assim para deitar e virar dos dois lados para descansar o corpo. (E5)

Olha, na verdade eu tenho dificuldade de manusear a bolsa, na limpeza dela, que são várias vezes ao dia, então meu marido aprendeu a mexer e troca para mim sempre. (E5)

A troca mesmo é duas vezes por semana, agora a limpeza a cada 4 h é minha esposa quem faz. (E4)

Minha irmã sempre me ajuda, coitada, ela me acompanha, quando eu to com dúvidas, ela me apoia. (E2)

Antes eu tinha vários lazeres, mas agora eu fico mais em casa, depois que coloquei a "sonda" eu me tornei uma pessoa mais caseira, nem na garagem eu ia. (E3)

Isso dificulta sair de casa e praticar atividades físicas, igual eu falei com o doutor. (E7) 
Hoje, estou doido para ficar livre dela (a bolsa), pra voltar às atividades normais, ela inibe realmente a gente de sair de casa. (E5)

Eu leio muita revista de oração, revistas de coisas que são boas para a saúde, eu faço um pouquinho de cada coisa. (E3)

Por enquanto não posso mais costurar né, mas quem sabe um dia, pra Deus nada é impossível! (E3)

Eu achei que era coisa de outro mundo, claro, chorei muito, mas o doutor disse que eu teria que usar, mas que eu poderia tirar algum dia, essa era a minha esperança. (E3)

Com o passar do tempo, eu mesmo buscava a bolsa no posto de saúde, eu mesma me virava, às vezes vinha umas bolsas grandes que eu não gostava, elas não paravam em mim, as meninas pediam umas pequenininhas que eu gostava, ai eu ficava mais feliz. (E3)

Nós não estamos tendo relação, depois que eu fui operada, eu fico com medo de manter relação, o médico até falou que podia. (E4)

Desde que iniciou o tratamento, não tenho mais vontade. (E5)

Hoje não tenho vontade de nada, não gosto de ficar acariciando ninguém, beijando ninguém, isso é péssimo, pois ele é carinhoso, eu que não sou. (E3)

Bom, no geral é a mesma coisa, não teve alteração não. (E4)

Geralmente uma vez por semana. (E4)

Assim, existe o desejo, a gente se beija, se abraça, se toca, só na hora da penetração que não acontece, mas a gente arruma um jeito de concluir o ato, porque você pode dar um jeito de concluir. (E5)

Então, assim, eu tenho vontade, sabe, ele também sente, procura, mas a gente fica com medo, coitado! (E2)
Meu marido não se importava, coisa mais engraçada, por isso que eu falo, ele é muito legal, ele é do bem mesmo. Tem homem que larga a mulher na hora se acontece um trem desses. (E3)

No relacionamento tem muita harmonia, meu marido diz que para ele... ele não é uma pessoa que vai ficar com outras mulheres, ele entende a situação que estou vivendo. (E2)

\section{DISCUSSÃO}

$\mathrm{Na}$ análise dos dados emergiram duas categorias temáticas: "enfrentamentos" e "sexualidade".

\section{Categoria 1: enfrentamentos}

A presença do estoma intestinal provoca diversas alterações na vida das pessoas que o têm, especialmente relacionadas à fisiologia gastrointestinal, imagem corporal e autoestima. O fornecimento de informações e orientações necessárias, o trabalho multidisciplinar, entre outros, são estratégias importantes a serem utilizadas para ajudar e apoiar os pacientes que se tornam colostomizados. Essas pessoas geralmente não estão preparadas para enfrentar essa nova condição em suas vidas. No entanto, apesar de não estarem preparadas inicialmente, após seu processo de adaptação como pessoas com estoma, elas começam a conceber a vida de outra maneira, especialmente quando a doença de base é o câncer ${ }^{11}$.

A maioria dos colostomizados definitivos demanda a utilização de uma bolsa coletora sobre o estoma para recebimento do efluente (fezes). Aqueles que não fazem uso se beneficiam da irrigação intestinal. Essa técnica requer habilidade motora e cognitiva, além de o paciente atender aos critérios de indicação.

O manejo do dispositivo coletor (bolsa) exige o esvaziamento de seu conteúdo, evitando o enchimento excessivo, o que pode resultar no descolamento do adesivo do sistema da bolsa, extravasamento do conteúdo fecal e, na maioria dos casos, danos da pele periestoma. É, portanto, crucial que paciente ou cuidador tenham clareza sobre quando e como esvaziar a bolsa coletora e como fazê-lo corretamente ${ }^{12}$.

A convivência com o dispositivo, gerando sentimentos conflituosos, preocupações e dificuldade para lidar com essa nova situação, também aparece em trabalho realizado no Nordeste do Brasil ${ }^{6}$. Os autores mencionam medo, vergonha e angústia, evidenciados pelos entrevistados de forma contundente. 
A revolta e a tristeza também foram recorrentes. Esses sentimentos foram identificados em estudo qualitativo sobre pessoas estomizadas ${ }^{4}$. Para os autores, o estado de tristeza parece ser inerente à condição de estomizado, pelo menos na etapa inicial.

Alguns entrevistados verbalizaram muita dificuldade de aceitação da condição de estomizado, inicialmente pela necessidade de lidar e manipular o estoma. Sua presença gera alteração física visível e significativa do corpo, que causa perda de sua integridade, dinamismo e autonomia, desencadeando conflitos pessoais e sociais, especialmente nas suas relações com o mundo exterior ${ }^{13}$.

A participação da família, especialmente do cônjuge, no processo de cuidado foi mencionada positivamente, o que sugere uma relação de companheirismo no cotidiano de alguns entrevistados. A inclusão de outros familiares emergiu como algo significativo nesse processo. A importância da família e dos amigos na reabilitação do colostomizado é mencionada em estudo sobre esse tema ${ }^{14}$. Porém, segundo os autores, nem sempre é simples envolver o familiar no cuidado a esse paciente, podendo surgir rejeição, medo e nojo frente ao primeiro contato com essa situação.

Foram recorrentes as citações sobre a dificuldade em sair de casa e a preferência por atividades caseiras. Em relação ao retorno ao trabalho, pesquisa sobre avaliação da educação em saúde recebida por colostomizados revelou incertezas e desconhecimento de como enfrentar o cotidiano, por ocasião do regresso às atividades diárias ${ }^{15}$. Segundo os autores, na dúvida, as pessoas pesquisadas preferiram limitar suas ações, tanto de convivência social quanto de atividades laborais.

A religiosidade foi tema recorrente. Observa-se que a crença em Deus e as orações emergiram em alguns relatos. Religiosidade, limitações advindas do processo de adoecer e o papel dela nesse percurso são descritos em trabalho qualitativo ${ }^{5}$ sobre pessoas estomizadas, no qual a religiosidade aparece como categoria. Os autores discorrem sobre a fragilidade e o envolvimento espiritual dos sujeitos pesquisados.

A questão da resiliência, presente em vários depoimentos, pode ser compreendida como um padrão de funcionamento de adaptação em relação aos riscos atuais e acumulados ao longo da vida. Abrange uma variedade de recursos psicológicos, necessários à superação de adversidades e ao controle interpessoal em interação com os apoios sociais ${ }^{16}$. Em alguns depoimentos foram evidenciados fatores que ajudaram direta ou indiretamente os sujeitos a atribuírem um novo sentido à adversidade vivenciada, seja por meio de competências individuais, culturais e familiares, olhar positivo como esperança e otimismo ou pela espiritualidade e transcendência.

\section{Categoria 2: sexualidade}

Durante o processo de entrevistas os participantes expressaram dificuldades na verbalização do assunto sexualidade. A impressão é de que abordagens inerentes ao tema são vistas pelos colostomizados como algo constrangedor. Conversar sobre sexualidade nessa condição é algo complexo de ser expresso ou exposto.

A desistência do ato sexual se mostrou recorrente. Parece decisão natural, por parte de alguns entrevistados, que a prática de relações sexuais se contrapõe à condição de estomizado.

A mudança física causada pela confecção do estoma pode afetar o desempenho sexual e a sexualidade do indivíduo. As dificuldades advêm das alterações na imagem corporal ou disfunções fisiológicas provenientes do próprio procedimento cirúrgico. O homem estomizado pode apresentar redução ou perda da libido, diminuição ou ausência da capacidade de ereção e alteração da ejaculação. As alterações mais frequentes apresentadas pela mulher são a redução ou perda da libido e dispareunia. Boa parte das dificuldades sexuais é de origem psicológica, sobretudo devido à vergonha frente ao parceiro e à sensação de estar sujo e repugnante, que resulta em medo de ser rejeitado ${ }^{17}$.

Existe ainda a possibilidade da existência de relacionamentos problemáticos anteriores à confecção do estoma ${ }^{18}$. Nesses relacionamentos, a situação de adoecimento e a confecção do estoma podem favorecer ou determinar o afastamento ou a interrupção da vivência das relações sexuais entre parceiros, justificada sob a alegação social de que uma pessoa "doente" não deve praticar o ato sexual, o que pode significar um grande alívio para alguns. Essa reflexão se faz necessária na abordagem da sexualidade das pessoas nessa condição.

Em contrapartida, a manutenção da atividade sexual após tornar-se colostomizado emergiu em alguns discursos. Revisão integrativa sobre sexualidade de estomizados ${ }^{19}$ obteve depoimentos de pessoas que não sentiram diferença na relação sexual, pois, segundo alguns relatos, o(a) parceiro(a) contribuiu nesse processo de adaptação.

Estudo realizado com 30 mulheres colostomizadas identificou como estratégia de enfretamento da sexualidade a retomada da relação sexual com algumas modificações para o repertório sexual, com utilização de técnica de gestão do estoma, tais como esvaziar o dispositivo antes da relação sexual e cobrir o dispositivo com diversos itens (toalhas, blusas, vestidos). A técnica de esconder o dispositivo tem a função de prevenir acidentes e escondê-la devido à sua própria repulsa ou do parceiro, já que existe uma aversão cultural às fezes e isso se torna incoerente com a excitação e com o desejo. 
Essas várias técnicas empregadas para esconder e gerenciar essa condição de ser colostomizada não só traduz a adaptabilidade e a criatividade dessas mulheres, mas também reflete a delicadeza e estética em relação aos seus corpos reconfigurados para retomarem sua sexualidade ${ }^{20}$.

A sexualidade ultrapassa a necessidade fisiológica e tem relação direta com a simbolização do desejo. Não se reduz aos genitais, refere-se à emoção que o sexo pode produzir, transcende definições físicas e se coloca como algo mais difuso, que permeia todos os momentos da vida, possui significados complexos, multifacetados e que concentram grande carga de subjetividade ${ }^{18}$.

Entretanto, alguns participantes do estudo relacionaram a sexualidade somente ao ato sexual quando abordam a vida íntima do casal. A sexualidade versus genitalidade é discorrida em artigo psicanalítico sobre sexualidade humana ${ }^{21}$. Para o autor, a visão puramente médica reduz a sexualidade apenas à genitalidade, circunscrevendo-a ao corpo biológico e ignorando a diferença entre ambas. Ele ainda refere que o corpo de que trata a psicanálise não é somente o biológico, mas sim o corpo erógeno, atravessado pela linguagem e inserido na trama de discursos que circulam no âmbito da cultura.

Alguns depoimentos sugerem uma relação de companheirismo. O companheirismo faz parte da vida de qualquer indivíduo e é influenciado por diversos fatores que contribuem para o bem-estar das pessoas. O enfrentamento da nova situação, de ser colostomizado, requer mudanças físicas e psicológicas, para que as rotinas sejam retomadas por eles e seus/suas companheiros $(\mathrm{as})^{19}$.

Os profissionais de saúde direcionam a assistência a aspectos relacionados a diagnóstico e tratamento biomédico, ou seja, voltada para a doença e sua cura. Os aspectos mais subjetivos, incluindo a sexualidade, são menos valorizados, dando a falsa impressão de que não fazem parte da saúde humana ${ }^{22}$.
Este estudo possibilitou desvelar os sentimentos de pessoas colostomizadas devido ao câncer e considera-se necessário revelar suas limitações, no que tange às especificidades dos participantes, por serem pessoas com cânceres nos estágios III e IV, e de suas realidades. Outra limitação foi o estudo ser restrito a um único serviço. Assim, os resultados encontrados não devem ser generalizados, porém, acredita-se que devam ser analisados com o intuito de fundamentar as ações de assistência a essa clientela. Dessa maneira, espera-se que esta pesquisa venha incentivar novos questionamentos que ampliem o conhecimento da temática apresentada.

\section{CONSIDERAÇÕES FINAIS}

Com este trabalho, foram percebidas as diferentes formas de adaptação a uma mesma situação e, por mais que a condição tenha sido relatada por todos como algo de difícil superação, foram elencadas peças-chaves que para alguns serviram como fatores protetivos.

A realização deste estudo permitiu obter informações e descortinar formas de enfrentamento do paciente oncológico quando se torna colostomizado. Nesse contexto, desvelaramse também importantes sentimentos e reações, somados ao diagnóstico de câncer, como lidar com o estoma e com as pessoas, fatores importantes para o desenvolvimento de programas de acolhimento a essa clientela.

Os resultados possibilitaram ainda reiterar que o processo de superação de um câncer e de adaptação após a confecção de um estoma é complexo, carregado de subjetividades e dificuldades, sendo que as interações com a família, os amigos e os profissionais de saúde podem auxiliar na retomada da autoestima.

\section{REFERÊNCIAS}

1. El-Shami K, Oeffinger KC, Erb NL, Willis A, Bretsch JK, Pratt-Chapman ML, et al. American Cancer Society Colorectal Cancer Survivorship Care Guidelines. CA Cancer J Clin. 2015:65(6):427-55.

2. De las Nieves $\mathrm{CB}$, Montoro $\mathrm{CH}$, Manãs $\mathrm{MC}$, Marin $\mathrm{CR}$, Crisol IS, Asencio JMM. Viviendo con un estoma digestivo: la importancia del apoyo familiar. Index Enferm. 2013;22(4):209-13.

3. Fernandes RM, Miguir ELB, Donoso TV. Perfil da clientela estomizada residente no município de Ponte Nova, Minas Gerais. Rev Bras Coloproctol. 2010;30(4):385-92.
4. Sales CA, Violin MR, Waidman MAP, Marcon SS, Silva MAP. Sentimentos de pessoas ostomizadas: compreensão existencial. Rev Esc Enferm USP. 2010;44(1):221-7.

5. Almeida SSL, Rezende AM, Schall VT, Modena CM. Os sentidos da corporeidade em ostomizados por câncer. Psicol Estud. 2010;15(4):761-9.

6. Batista MRFF, Rocha FCV, Silva DMG, Silva Junior FJG. Autoimagem de clientes com colostomia em relação à bolsa coletora. Rev Bras Enferm. 2011;64(6):1043-7. 
7. Cetolin SF, Beltrame V, Cetolin SK, Presta AA. Dinâmica sócio-familiar com pacientes portadores de ostomia intestinal definitiva. ABCD Arq Bras Cir Dig. 2013;26(3):170-2.

8. Vieira LM, Ribeiro BNO, Gatti MAN, Simeão SFAP, Conti MHS, Vitta A. Câncer colorretal: entre o sofrimento e o repensar na vida. Saúde Debate. 2013;37(97):261-9.

9. Dessote CAM, Teixeira ASM, Sousa CDA, Sonobe HM. Estratégias de ensino em enfermagem perioperatória: uma avaliação discente. Rev SOBECC. 2015;20(4):189-96.

10. Bardin L. Análise de conteúdo. 5 ed. Lisboa: Edições 70; 2011.

11. Backes MTS, Backes DS, Erdmann AL. Feelings and expectations of permanent colostomy patients. J Nurs Educ Pract. 2012;2(3):9-14.

12. Colwell JC, Kupsick PT, McNichol LL. Outcome criteria for discharging the patient withanewostomy from home healthcare: aWOCNSociety Consensus Conference. J Wound Ostomy Continence Nurs. 2016;43(3):269-73.

13. Cardoso DBR, Almeida CE, Santana ME, Carvalho DS, Sonobe HM, Sawada NO. Sexualidade de pessoas com estomias intestinais. Rev Rene. 2015;16(4):576-85.

14. Ardigo FS, Amante LN. Conhecimento do profissional acerca do cuidado de enfermagem à pessoa com estomia intestinal e família. Texto Contexto - Enferm. 2013;22(4):1064-71.
15. Klein DP, Silva DMGF. Avaliação da educação em saúde recebida pela pessoa com estoma intestinal na perspectiva da clínica ampliada. Ciênc Cuid Saúde. 2014;13(2):262-70.

16. Fontes AP, Néri AL. Resiliência e velhice: revisão de literatura. Ciênc Saúde Colet. 2015;20(5):1475-95.

17. Borwell B. Rehabilitation and stoma care: addressing the psychological needs. Br J Nurs. 2009;18(4):S20-5.

18. Paula MAB, Takahashi RF, Paula PR. Os significados da sexualidade para a pessoa com estoma intestinal definitivo. Rev Bras Colo-proctol. 2009;29(1):77-82.

19. Barreto APC, Valença MP. A sexualidade do paciente estomizado: revisão integrativa. Rev Enferm UFPE on line. 2013;7(esp):4935-43.

20. Ramirez M, McMullen C, Grant M, Altdchuler A, Hornbrook MC, Krouse RS. Figuring out sex in a reconfigured body: experiences of female colorectal cancer survivors with ostomies. Women Health. 2009;49(8):608-24.

21. Couto OHC. Tudo azul com o sexual? Viagra e sexualidade. Reverso. 2011;33(61):83-9.

22. Martins W, Penna LHG, Paula MAB, Pereira CDC, Leite HC. Sexualidade, estoma e gênero: revisão integrativa da literatura. Rev Estima. 2011;9(1):39-46. 